Canadian

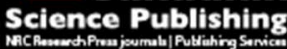

Applied Physiology, Nutrition, and Metabolism Physiologie appliquée, nutrition et métabolisme

\title{
Regenerated soleus muscle shows reduced creatine kinase efflux after contractile activity in vitro
}

\begin{tabular}{|r|l|}
\hline Journal: & Applied Physiology, Nutrition, and Metabolism \\
\hline Manuscript ID: & apnm-2014-0274.R1 \\
\hline Manuscript Type: & Article \\
\hline Date Submitted by the Author: & n/a \\
\hline Complete List of Authors: & $\begin{array}{l}\text { Baltusnikas, Juozas; Lithuanian Sports University, } \\
\text { Kilikevicius, Audrius; Lithuanian Sports University, } \\
\text { Venckunas, Tomas; Lithuanian Sports University, } \\
\text { Fokin, Andrej; Lithuanian Sports University, } \\
\text { Lionikas, Arimantas; University of Aberdeen, } \\
\text { Ratkevicius, Aivaras; University of Aberdeen, ; Lithuanian Sports } \\
\text { University, }\end{array}$ \\
\hline Keyword: & muscle damage < muscle \\
\hline &
\end{tabular}


1 Regenerated soleus muscle shows reduced creatine kinase efflux after contractile activity in

2 vitro

3

4 Baltusnikas Juozas ${ }^{1}$, Kilikevicius Audrius ${ }^{1}$, Venckunas Tomas ${ }^{1}$, Fokin Andrej ${ }^{1}$, Lionikas

5 Arimantas $^{2}$, Ratkevicius Aivaras ${ }^{1,2}$.

$6{ }^{1}$ Institute of Sports Sciences and Innovation, Lithuanian Sports University, Sporto 6, LT-44221,

7 Kaunas, Lithuania;

$8{ }^{2}$ School of Medical Sciences, University of Aberdeen, King's College AB24 3FX, Aberdeen,

9 Scotland, UK.

11 Correspondence to:

12 Juozas Baltušnikas, MSc

13 Institute of Sports Sciences and Innovation

14 Lithuanian Sports University, Sporto 6, LT-44221, Kaunas, Lithuania

15 Phone: +370 671 00819; Fax: +370 37204515

16 E-mail: juozas.baltusnikas@1su.1t

18 E-mails:

19 Kilikevicius Audrius: audrius.kilikevicius@1su.lt

20 Venckunas Tomas: tomas.venckunas@1su.1t

21 Fokin Andrej: fokinandrej@yahoo.com

22 Lionikas Arimantas: a.lionikas@abdn.ac.uk

23 Ratkevicius Aivaras: a.ratkevicius@abdn.ac.uk 


\section{Abstract}

26 Regenerated skeletal muscles show less muscle damage after strenuous muscle exercise. The aim

27 of the studies was to investigate if the regeneration is associated with reduced muscle creatine

28 kinase $(\mathrm{CK})$ efflux immediately after the exercise. Cryolesion was applied to the soleus muscle

29 (SOL) of 3 month old C57BL/6J male mice. Then total CK efflux was assessed in vitro in the

30 regenerated (REG) muscles without exercise or after 100 eccentric contractions. The same

31 measurements were performed in the control (CON) muscles which were not exposed to

32 cryolesion. REG muscles generated weaker $(\mathrm{P}<0.05)$ twitches, but stronger $(\mathrm{P}<0.05) 150-\mathrm{Hz}$

33 and $300-\mathrm{Hz}$ tetani with prolonged $(\mathrm{P}<0.01)$ contraction times compared to the control muscles.

34 There was no difference between REG and CON muscles in the total CK efflux without exercise,

35 but only CON muscles showed an increase $(\mathrm{P}<0.001)$ in the $\mathrm{CK}$ efflux after the exercise. Our

36 results suggest that muscle regeneration is associated with modulation of contractile properties

37 and improvement in muscle resistance to damage after eccentric exercise.

39 Keywords: cryolesion, primary damage, muscle damage, repeated bout effect, eccentric 40 exercise, lengthening contractions, mice. 


\section{Introduction}

44 Efflux of creatine kinase (CK) from skeletal muscles is a popular marker of muscle damage after 45 exercise and disease (Brancaccio et al. 2007). It is often assessed by measuring plasma CK 46 activity. It has been known for some time that eccentric exercise is associated with a particular

47 large increase in plasma CK compared to other types of contractile activity (Newham et al. 48 1986). Plasma $\mathrm{CK}$ and other indicators of muscle damage show ameliorated response to a 49 repeated bout of exercise even if it is performed several weeks after the first exercise bout 50 (Clarkson et al. 1992). The mechanisms underlying this repeated bout effect remain unclear.

52 Eccentric contractions can induce disruption of myofibrils and thus cause a prolonged 53 impairment in muscle force generating capacity (McHugh 2003). The reasons for CK efflux from 54 skeletal muscles are controversial since muscle exercise might promote an increase in 55 permeability of muscle fibers which is not necessarily associated with damage to muscle fibers

56 (Yu et al. 2013). It is believed that inflammatory cell infiltration of skeletal muscles can also

57 promote secondary muscle damage after exercise and thus contribute to muscle CK efflux after 58 exercise (McHugh 2003; Tidball 2011). It is important to examine effects of primary muscle 59 damage during exercise and secondary damage after exercise separately in order to clarify the 60 mechanisms of repeated bout effect. Reduction in primary muscle damage would reflect 61 increased resistance of muscle structures to disruption during exercise while modulation of the 62 inflammatory responses would determine the secondary damage. Indeed, it is unclear if the 63 increase in plasma $\mathrm{CK}$ activity after exercise is due to the primary or secondary muscle damage.

64 There is often no or only a minor increase in plasma CK activity immediately after exercise and 65 plasma CK peaks 1-3 days after the exercise coinciding with the peak in muscle soreness 
66 (Armstrong 1984; Fredsted et al. 2008). Isolated skeletal muscles in vitro are well suited for

67 studying primary muscle damage since measurements of muscle CK efflux can be performed

68 immediately after exercise with limited contribution of the secondary muscle damage. Mouse

69 soleus muscle (SOL) provides a good model for such studies since it contains approximately

70 equal proportions of type I and type II fibres type (Kilikevicius et al. 2013; Denies et al. 2014)

71 and thus resembles human quadriceps muscle, which is often used in human studies (Staron et al.

72 2000).

73

74 Muscle incubation with damaging agents leads to injury and subsequent regeneration of rat

75 skeletal muscles (Jackson et al. 1987). In another study the regenerated extensor digitorum

76 longus muscle of rats showed reduced ultrastructural damage compared to the control muscle

77 when examined 3 days after plyometric exercise (Devor and Faulkner 1999). It is, however,

78 unclear if this apparent resistance to muscle damage was caused by modulation of primary or

79 secondary mechanisms of muscle damage. The aim of our study was to test the hypothesis that

80 muscle regeneration after cryolesion is associated with a reduction in muscle CK efflux

81 immediately after exercise and could be attributed to reduction in the primary muscle damage.

82 We compared CK efflux from the regenerated and control SOL in vitro without any prior

83 exercise and immediately after repeated eccentric contractions.

\section{$85 \quad$ Methods}

86 Animals and experiments; All procedures involving mice were approved by the Lithuanian 87 Republic Alimentary and Veterinary Public Office (Nr. 0223). As in our previous studies, $88 \mathrm{C} 57 \mathrm{BL} / 6 \mathrm{~J}$ mice were housed in the standard cages without exercise equipment, one to three mice 
89 per cage at a temperature of $22-24^{\circ} \mathrm{C}$ and $40-60 \%$ humidity (Ratkevicius et al. 2010; 90 Kilikevicius et al. 2013) with the normal 12/12-h light/dark cycle. Animals were fed a standard 91 chow diet $(76.9 \% \mathrm{kcal}$ from carbohydrate, $16.9 \%$ kcal from protein, $6.2 \% \mathrm{kcal}$ from fat;

92 Kedainiu grudai, Kedainiai, Lithuania) and received tap water ad libitum. As described below 93 (see "Muscle contractile properties and CK efflux"), we assessed CK efflux from SOL muscle at 94 rest without any prior exercise $(n=6)$ and after 100 eccentric contractions $(n=8)$ in 12 week old 95 male mice. These muscles are referred to as control $(\mathrm{CON})$ muscles. We have also performed the 96 same measurements at rest $(n=9)$ and after exercise $(n=9)$ in SOL after regeneration and refer 97 to these muscles as regenerated (REG). In a separate series of experiments, we have also 98 assessed effects of two-hour in vitro incubation on peak isometric force of CON muscles $(\mathrm{n}=4)$ 99 using the same procedures described for the assessment of muscle CK efflux.

100 Muscle regeneration; Muscle regeneration was induced by cryolesion as described elsewhere 101 (Irintchev et al. 2002). Briefly, at age of 2 months male mice $(n=18)$ were anesthetized by 102 intraperitoneal injection of the anesthetics: ketamine (120 mg/kg; Richter Pharma AG, Wels, 103 Austria) and xylazine (14 mg/kg; Eurovet Animal Health B.V., Bladel, Netherlands). The hair 104 from the leg was removed using electric shaver. SOL was exposed by making the incision 105 through the overlaying skin and connective tissue, and retracting the adjacent gastrocnemius 106 muscle. Muscle cryolesion was induced by touching the middle portion of SOL with flat end of a 107 copper rod $(3 \times 0.7 \mathrm{~mm})$ precooled in liquid nitrogen and maintaining its position for $5 \mathrm{~s}$. After 2 108 min when the muscles had thawed, the skin incision was closed with polyamide threads (4-0 109 Ethilon; Ethicon, Norderstedt, Germany) and mice were placed on $37^{\circ} \mathrm{C}$ temperature plate for 110 several hours to avoid hypothermia. After 29 days (at age of $\sim 3$ months) we assessed contractile 111 properties and CK efflux. 
112 Muscle contractile properties and CK efflux; Mice were euthanized by cervical dislocation

113 and weighted immediately afterwards (Kern, ABS 80-4, Balingen, Germany). Sutures were

114 attached to the proximal and distal tendons of SOL from left leg. The muscle was then excised

115 and fixed between two platinum plate electrodes in $50 \mathrm{ml}$ Radnotti tissue bath filled with Tyrode

116 solution (121 mM NaCl, $5 \mathrm{mM} \mathrm{KCl}, 0.5 \mathrm{mM} \mathrm{MgCl}_{2}, 1.8 \mathrm{mM} \mathrm{CaCl}_{2}, 0.4 \mathrm{mM} \mathrm{NaH} \mathrm{PO}_{4}, 0.1 \mathrm{mM}$

117 EDTA, $24 \mathrm{mM} \mathrm{NaHCO}_{3}, 5.5 \mathrm{mM}$ glucose, $\mathrm{pH}$ adjusted to 7.4) bubbled with $95 \% \mathrm{O} 2$ and $5 \%$

$118 \mathrm{CO} 2$. The distal tendon of the muscle was attached to a hook and the proximal end was tied

119 directly to the lever of muscle test system (1200A-LR Muscle Test System, Aurora Scientific

120 Inc., Aurora, Canada). The muscle was then left to equilibrate in the solution for $7 \mathrm{~min}$.

121 Afterwards muscle length was increased in steps every $2 \mathrm{~min}$ and the muscle was stimulated at

$122150 \mathrm{~Hz}$ for $3 \mathrm{~s}$. This procedure was continued until no further increase in muscle force was seen

123 with the increase in muscle length. Thereafter the muscle was photographed with the length scale

124 in the background to assess muscle length with a precision of $0.5 \mathrm{~mm}$. The subsequent force

125 measurements were performed at this optimal muscle length. Firstly, single twitch was evoked.

126 Then force frequency relationship was determined by stimulating muscle at 30, 50, 75, 150, 300

$127 \mathrm{~Hz}$ for $3 \mathrm{~s}$ with 2 min intervals in between the stimuli trains. Afterwards, SOL was subjected to

128 repeated eccentric contractions every $10 \mathrm{~s}$ or the control experiment without exercise. For the

129 eccentric exercise, SOL was stimulated at $150 \mathrm{~Hz}$ stimulation for $700 \mathrm{~ms}$. Over the last $200 \mathrm{~ms}$

130 of this stimulation $3.5 \mathrm{~mm}$ ramp stretch was performed followed by $200 \mathrm{~ms}$ gradual return of the

131 muscle to the initial length without any stimulation. In the control experiment, SOL was left at

132 rest for $\sim 20 \mathrm{~min}$. After the exercise or the control experiment, SOL was placed in $2 \mathrm{ml}$ of

133 Tyrode solution for 2 hours. Afterwards, muscles were dried and weighed. $250 \mu$ l of Tyrode

134 solution was taken to assess CK activity with biochemical analyzer (Spotchem ${ }^{\mathrm{TM}}$ EZ SP-4430, 
135 Menarini Diagnostics, Winnersh-Wokingham, UK) with soft reagent strips (ARKRAY Factory, 136 Inc., Shiga, Japan).

137 Statistical analysis; All data analysis was performed using Prism 5.0 software. The two factor 138 analysis of variance (ANOVA) was used to assess effects of regeneration and exercise on muscle 139 contractile properties and muscle CK efflux. The post hoc testing was carried out using t-tests 140 with a Bonferroni correction for multiple comparisons. Person's correlation coefficient was 141 calculated to investigate the association between the variables. All the tests were two-tailed with 142 significance level was set at $\mathrm{P}<0.05$.

\section{Results}

145 The data on body mass, muscle mass, tetanic force, specific force and optimal muscle length of 146 CON and REG muscles are presented in Table 1. There were no differences between CON and 147 REG muscles in these parameters.

149 Data on force-frequency relationship as well as twitch and tetanus properties are shown in Fig. 1.

150 In comparison to CON muscles, REG muscles generated relatively less $(\mathrm{P}<0.01)$ force in single

151 twitches and more force $(\mathrm{P}<0.001)$ in tetani at $150 \mathrm{~Hz}$ and $300 \mathrm{~Hz}$. Twitch contraction times did 152 not differ between REG and CON muscles, but REG muscles showed shorter $(\mathrm{P}<0.001)$

153 relaxation times than $\mathrm{CON}$ muscles. On the other hand, REG muscles had prolonged $(\mathrm{P}<0.01)$ 154 contraction time in $150 \mathrm{~Hz}$ tetanus compared to CON muscles.

156 Data on peak isometric and eccentric force during eccentric exercise are shown in Fig. 2. Peak 157 isometric force decreased more than eccentric force during the exercise $(\mathrm{P}<0.001)$. REG 
158 muscles tended to maintain isometric force better than CON muscles though the difference was

159 not significant. There were no differences between CON and REG muscles in eccentric force.

161 A separate set of experiments on control SOL showed that peak isometric force decreased $(\mathrm{P}<$ 1620.05 ) by $6.9 \pm 2.8 \%$ when muscles were incubated for 2 hours in Tyrode solution as during the 163 assessment of muscle CK efflux. Data on the total muscle CK efflux without any prior exercise 164 and after 100 repeated contractions are shown in Fig. 3. The CK efflux did not differ between 165 REG and CON muscles when muscles were not subjected to prior exercise. However, the total 166 muscle $\mathrm{CK}$ efflux was significantly $(\mathrm{P}<0.001)$ larger in the exercised CON compared to 167 exercised REG muscles and the non-exercised muscles. The exercised REG muscles did not 168 differ from the non-exercised muscles. The total muscle CK efflux did not correlate with force 169 loss by the end of exercise in CON muscles, but the there was a positive correlation between 170 these parameters in REG muscles.

171

\section{Discussion}

173 The main aim of the study was to test the hypothesis that muscle regeneration is associated with

174 a reduction in exercise-induced increase in the total muscle CK efflux. Our results show that the

175 regenerated muscles produced weaker single twitches, but stronger tetani with longer contraction 176 times compared to the control muscles. The regenerated muscles did not differ from the control 177 muscles in the total CK efflux without exposure to exercise. However, in contrast to the control 178 muscles, these muscles did not show any increase in the total CK efflux after the repeated 179 eccentric contractions. These results suggest that muscle regeneration is associated with 
180 modulation of contractile properties and increased resistance to loss of muscle proteins during 181 exercise.

183 Myotoxin (bupivacaine) injection has been often applied to induce muscle injury with 184 subsequent regeneration (Devor and Faulkner 1999). Muscle cryolesion induces a similar 185 phenomenon (Irintchev et al. 2002; Pereira et al. 2014). There were no differences in either body 186 mass or muscle mass between the mice exposed to muscle cryolesion and the control mice in our 187 study. Peak tetanic force, specific force and optimal length were also similar in the regenerated 188 and control muscles. Thus mice and treated muscles recovered fully within 29 days after the 189 cryolesion. However, the regenerated muscles showed slow rate of force generation in tetani. 190 Indeed, muscle regeneration is associated with a shift towards slower muscle fiber types and 191 myosin isoforms (Whalen et al. 1990; Irintchev et al. 2002). These changes might contribute to 192 the lower rate of tetanic force generation and a tendency towards better force maintenance in 193 repeated contractions. A robust contractile response of regenerated muscle suggests a full 194 recovery from the damage caused by the intervention. This would be consistent with earlier 195 findings showing that the number of fibres do not decline in the muscle after the cryolesion 196 (Irintchev et al. 2002). However, it is unclear what mechanisms are responsible for the 197 improvement in force output at high frequencies of electrical stimulation coupled with small 198 amplitude and fast relaxation of single twitches. This might be due to alteration in intracellular 199 calcium handling, but changes in muscle fibre force summation are also possible. It appears that 200 the total number of muscle fibres tends to increase in the regenerated muscles after the 201 cryolesion (Irintchev et al. 2002). However, it is unclear if all these fibres can contribute equally 202 to force output at different frequencies of electrical stimulation. Assessment of glycogen 
203 depletion patterns in muscle fibres after electrical stimulation might be a useful experimental

204 strategy to resolve this uncertainty in future studies.

205 We used a precooled copper rod to induce muscle cryolesion. This procedure followed by 206 subsequent muscle regeneration might have resulted in altered permeability of sarcolemma.

207 However, our results are inconsistent with such scenario. The total CK efflux at rest, when

208 muscles were not subjected to exercise, did not differ between the regenerated and control

209 muscles. The magnitude of this CK efflux was also similar to the previously reported for rat

210 soleus muscle in vitro (Jackson et al. 1987). Thus it is unlikely that there were significant

211 differences in muscle membrane permeability to CK molecules between the control and

212 regenerated muscles.

213 We assessed the total CK efflux during the two-hour muscle incubation in Tyrode solution.

214 Consistent with previous studies (Plant et al. 2001), peak force generating capacity showed only

215 a small decline during the two-hour muscle incubation which suggests that there was no major

216 disruption of muscle contractile apparatus. We did observe a tendency for an increase in muscle

217 mass after this procedure (unpublished observation). This might be a reflection of an increase in

218 muscle water content (Sjøgaard et al, 1985), and it is likely that muscle CK efflux is partially

219 associated with the osmotic stress generated by muscle incubation in Tyrode buffer. However,

220 skeletal muscles are well adapted to withstand such stresses as there is an increase in the

221 muscle's extracellular and intracellular water content after exercise of submaximal and maximal

222 intensity, respectively (Sjøgaard et al, 1985). Our results suggest, however, that muscle

223 regeneration is not associated with increased muscle resistance to CK efflux at rest under the

224 influence of mild osmotic stress. 
226 There were significant differences between the control and regenerated muscles after the

227 exercise. The regenerated muscles showed no increase in CK efflux whereas the control muscles

228 showed a substantial, 2.8-fold, increase. These differences between the regenerated and control

229 muscles could not be explained by the variation in the mechanical stresses experienced by

230 muscles since the regenerated muscles produced more force than control muscles during the

231 exercise. Thus, regenerated muscles showed a true increase in resistance to exercise-induced

232 muscle CK efflux. Interestingly, CK efflux from the regenerated muscles correlated with force

233 loss during the exercise, but there was no such correlation for the control muscles (see Fig. 3).

234 This suggests that there was a qualitative difference between the muscles. It is likely that a

235 disruption of muscle structure is needed to cause a significant increase in CK efflux from the

236 regenerated muscles while the control muscles show an increase in the muscle CK efflux after

237 exercise even without damage to the contractile machinery. Indeed, skeletal muscles often show

238 a significant CK loss even when there are no clear signs of the ultrastructural damage (Yu et al.

239 2013).

241 It is often argued that exercise training can lead to an increase muscle collagen content which

242 might affect mechanical properties and thus improves muscle resistance to exercise-induced

243 muscle damage (McHugh 2003; Mackey et al. 2004). Indeed, eccentric exercise training can lead

244 to an increase in dynamic and passive stiffness of skeletal muscles (Reich et al. 2000). However,

245 we did not observe any difference between the regenerate and control muscles in forces

246 generated during the eccentric phase of the contractions when the controlled stretching of the

247 muscles was imposed. These findings speak against muscle stiffness being of importance for 248 exercise-induced muscle $\mathrm{CK}$ efflux in the regenerated muscles. A shift in muscle fiber 
249 composition towards slower contraction muscle fibers and myosin isoforms could be of greater

250 importance. Slow twitch muscle fibers show less damage than fast twitch fibers after exercise

251 (Chapman et al. 2013).

252

253 Our findings agree with previous studies on rat muscles showing less structural damage in

254 regenerated muscles 3 days after plyometric exercise (Devor and Faulkner, 1999). Our results

255 suggest that reduced primary muscle damage is likely to be a major factor in regeneration-

256 induced resistance to muscle damage. It appears that stimulation of muscle regeneration might be

257 a useful strategy in increasing resistance to exercise-induced muscle damage. Leucine

258 supplementation increased the gain in myofiber size during regeneration though its effects on

259 muscle resistance to exercise-induced damage are less clear (Pereira et al. 2014).

260

261 In summary, our results show that muscle regeneration is associated with modulation of

262 contractile properties and increased resistance to the primary muscle damage during exercise, but

263 it is not protecting against muscle CK efflux at rest when mild osmotic stresses are applied. 


\section{Acknowledgements}

266 We are very grateful to Petras Jeneckas and Audrius Capskas for technical assistance. The study 267 was supported by a grant (No. MIP-067/2012) from the Research Council of Lithuania.

\section{References}

270 Armstrong, R.B. 1984. Mechanisms of exercise-induced delayed onset muscular soreness: a brief 271 review. Med. Sci. Sports Exerc. 16(6): 529-38.

272 Brancaccio, P., Maffulli, N., and Limongelli, F.M. 2007. Creatine kinase monitoring in sport 273 medicine. Br. Med. Bull. 81-82: 209-30.

274 Chapman, D.W., Simpson, J.A., Iscoe, S., Robins, T., and Nosaka, K. 2013. Changes in serum 275 fast and slow skeletal troponin I concentration following maximal eccentric contractions. J. Sci. 276 Med. Sport, 16(1): 82-5. doi:10.1016/j.jsams.2012.05.006.

277 Clarkson, P.M., Nosaka, K., and Braun, B. 1992. Muscle function after exercise-induced muscle 278 damage and rapid adaptation. Med. Sci. Sports Exerc. 24(5): 512-20.

279 Denies, M.S., Johnson, J., Maliphol, A.B., Bruno, M., Kim, A., Rizvi, A., Rustici, K., and 280 Medler, S. 2014. Diet-induced obesity alters skeletal muscle fiber types of male but not female 281 mice. Physiol. Rep. 2(1):e00204. doi:10.1002/phy2.204.

282 Devor, S.T., and Faulkner, J.A. 1999. Regeneration of new fibers in muscles of old rats reduces 283 contraction-induced injury. J. Appl. Physiol. 87(2): 750-6.

284 Fredsted, A., Clausen, T., and Overgaard, K. 2008. Effects of step exercise on muscle damage 285 and muscle Ca2+ content in men and women. J. Strength Cond. Res. 22(4): 1136-46. 286 doi:10.1519/JSC.0b013e318173db9b. 
287 Irintchev, A., Zweyer, M., Cooper, R.N., Butler-Browne, G.S., and Wernig, A. 2002. Contractile 288 properties, structure and fiber phenotype of intact and regenerating slow-twitch muscles of mice 289 treated with cyclosporin A. Cell Tissue Res. 308(1): 143-56.

290 Jackson, M.J., Wagenmakers, A.J., Edwards, R.H. 1987. Effect of inhibitors of arachidonic acid 291 metabolism on efflux of intracellular enzymes from skeletal muscle following experimental 292 damage. Biochem. J. 241(2):403-407.

293 Kilikevicius, A., Venckunas, T., Zelniene, R., Carroll, A.M., Lionikaite, S., Ratkevicius, A., and 294 Lionikas, A. 2013. Divergent physiological characteristics and responses to endurance training 295 among inbred mouse strains. Scand. J. Med. Sci. Sports, 23(5): 657-68. doi:10.1111/j.1600$296 \quad 0838.2012 .01451$.

297 Mackey, A.L., Donnelly A.E., Turpeenniemi-Hujanen, T., and Roper, H.P. 2004. Skeletal 298 muscle collagen content in humans after high-force eccentric contractions. J. Appl. Physiol. 299 97(1):197-203.

300 Mair, J., Mayr, M., Müller, E., Koller, A., Haid, C., Artner-Dworzak, E., Calzolari, C., Larue, C., 301 and Puschendorf, B. 1995. Rapid adaptation to eccentric exercise-induced muscle damage. Int. J. 302 Sports Med. 16(6): 352-6.

303 McHugh, M.P. 2003. Recent advances in the understanding of the repeated bout effect: the 304 protective effect against muscle damage from a single bout of eccentric exercise. Scand. J. Med. 305 Sci. Sports, 13(2): 88-97.

306 Newham, D.J., Jones, D.A., Edwards, R.H. 1986. Plasma creatine kinase changes after eccentric 307 and concentric contractions. Muscle Nerve, 9(1):59-63. 
308 Pereira, M.G., Baptista, I.L., Carlassara, E.O., Moriscot, A.S., Aoki, M.S., Miyabara, E.H. 2014.

309 Leucine supplementation improves skeletal muscle regeneration after cryolesion in rats. PLoS

310 One, 9(1):e85283. doi: 10.1371/journal.pone.0085283.

311 Pizza, F.X., Davis, B.H., Henrickson, S.D., Mitchell, J.B., Pace, J.F., Bigelow, N., DiLauro, P.,

312 and Naglieri, T. 1996. Adaptation to eccentric exercise: effect on CD64 and CD11b/CD18

313 expression. J. Appl. Physiol. 80(1):47-55.

314 Plant, D.R., Gregorevic, P., Williams, D.A., Lynch, G.S. 2001. Redox modulation of maximum

315 force production of fast-and slow-twitch skeletal muscles of rats and mice. J. Appl. Physiol. $31690(3): 832-8$

317 Ratkevicius, A., Carroll, A.M., Kilikevicius, A., Venckunas, T., McDermott, K.T., Gray, S.R., 318 Wackerhage, H., and Lionikas, A. 2010. H55N polymorphism as a likely cause of variation in 319 citrate synthase activity of mouse skeletal muscle. Physiol. Genomics, 42A(2): 96-102.

320 doi:10.1152/physiolgenomics.00066.2010.

321 Reich, T.E., Lindstedt, S.L., LaStayo, P.C., and Pierotti, D.J. 2000. Is the spring quality of

322 muscle plastic? Am. J. Physiol. Regul. Integr. Comp. Physiol. 278(6): R1661-6.

323 Sjøgaard, G., Adams, R.P., Saltin, B. 1985. Water and ion shifts in skeletal muscle of humans 324 with intense dynamic knee extension. Am. J. Physiol. 248(2):R190-6.

325 Staron, R.S., Hagerman, F.C., Hikida, R.S., Murray, T.F., Hostler, D.P., Crill, M.T., Ragg, K.E., 326 and Toma, K. 2000. Fiber type composition of the vastus lateralis muscle of young men and 327 women. J. Histochem. Cytochem. 48(5): 623-9.

328 Tidball, J.G. 2011. Mechanisms of muscle injury, repair, and regeneration. Compr. Physiol. 1(4):

329 2029-62. doi:10.1002/cphy.c100092. 
330 Whalen, R.G., Harris, J.B., Butler-Browne, G.S., Sesodia, S. 1990. Expression of myosin 331 isoforms during notexin-induced regeneration of rat soleus muscles. Dev. Biol. 141(1):24-40.

332 Yu, J.G., Liu, J.X., Carlsson, L., Thornell, L.E., Stål, P.S. 2013. Re-evaluation of sarcolemma 333 injury and muscle swelling in human skeletal muscles after eccentric exercise. PLoS One, 334 8(4):e62056. doi:10.1371/journal.pone.0062056.

335 
336 Table 1. Body mass as well as soleus (SOL) muscle mass, peak tetanic force, specific force and

337 optimal muscle length in mice with the control (CON) and regenerated (REG) muscles. Values

338 are means \pm S.D.

\begin{tabular}{|c|c|c|c|c|c|}
\hline & $\begin{array}{c}\text { Body mass } \\
(\mathrm{g})\end{array}$ & $\begin{array}{c}\text { Soleus mass } \\
(\mathrm{mg})\end{array}$ & $\begin{array}{c}\text { Tetanic force } \\
(\mathrm{mN})\end{array}$ & $\begin{array}{c}\text { Specific force } \\
(\mathrm{N} / \mathrm{g} \text { muscle })\end{array}$ & $\begin{array}{c}\text { Optimal } \\
\text { muscle length } \\
(\mathrm{mm})\end{array}$ \\
\hline CON & $24.6 \pm 1.9$ & $10.0 \pm 1.4$ & $166.2 \pm 19.4$ & $18.7 \pm 2.3$ & $16.2 \pm 1.6$ \\
\hline REG & $23.8 \pm 1.2$ & $9.1 \pm 0.7$ & $175.6 \pm 23.2$ & $19.2 \pm 1.7$ & $15.8 \pm 0.8$ \\
\hline
\end{tabular}

339

340 
$341 \quad$ Figure 1.
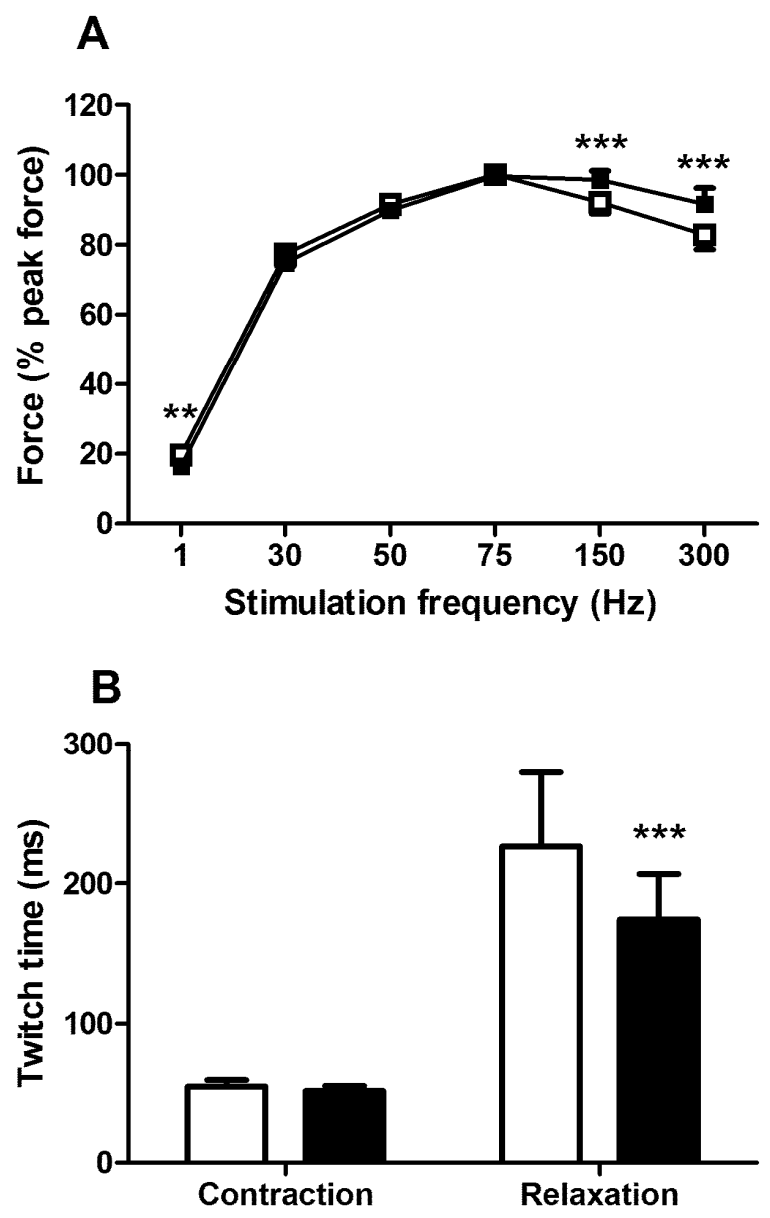

C

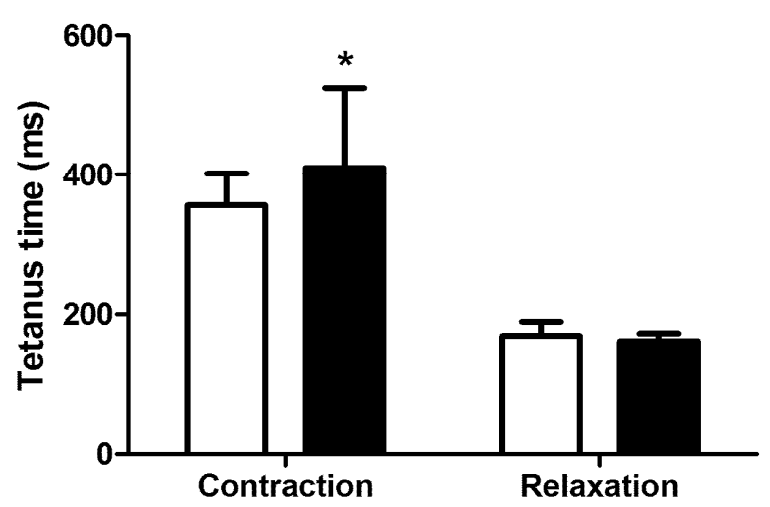




\section{Figure 2.}

A

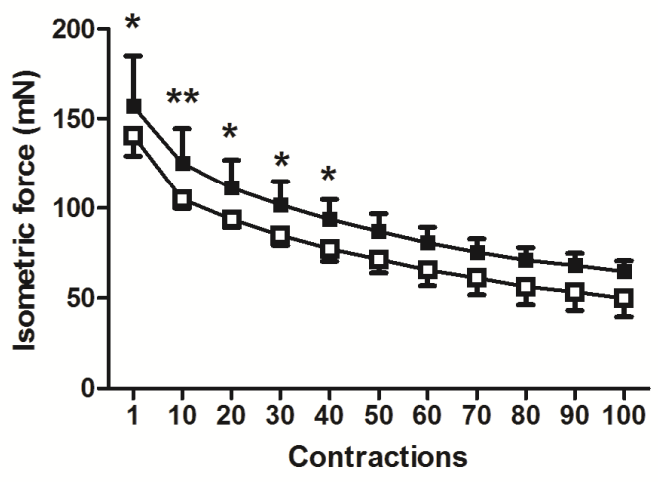

C

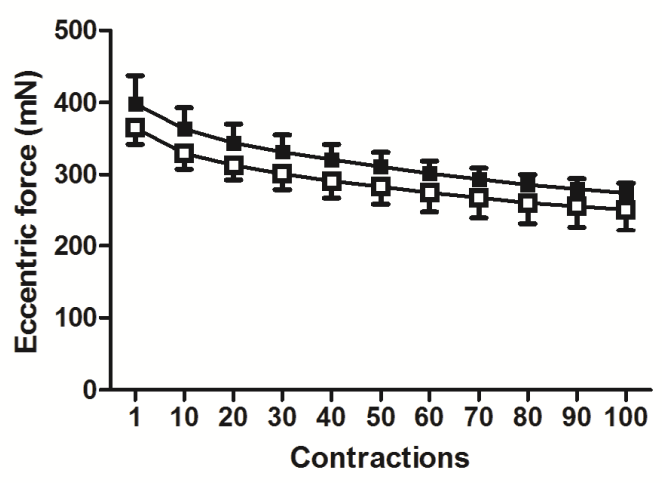

B

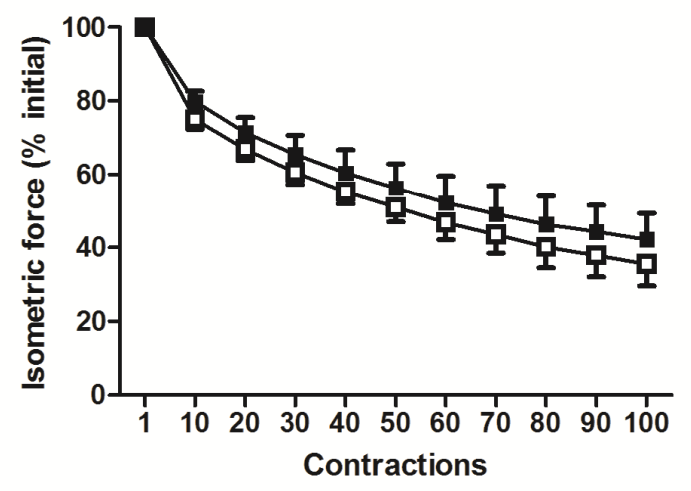

D

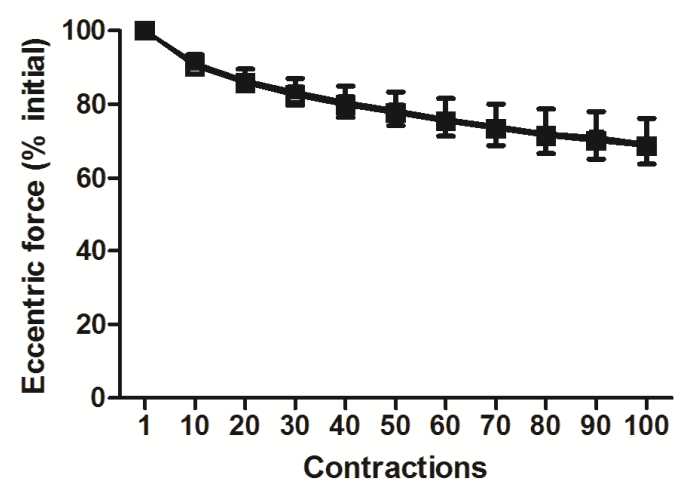


$347 \quad$ Figure 3.

A

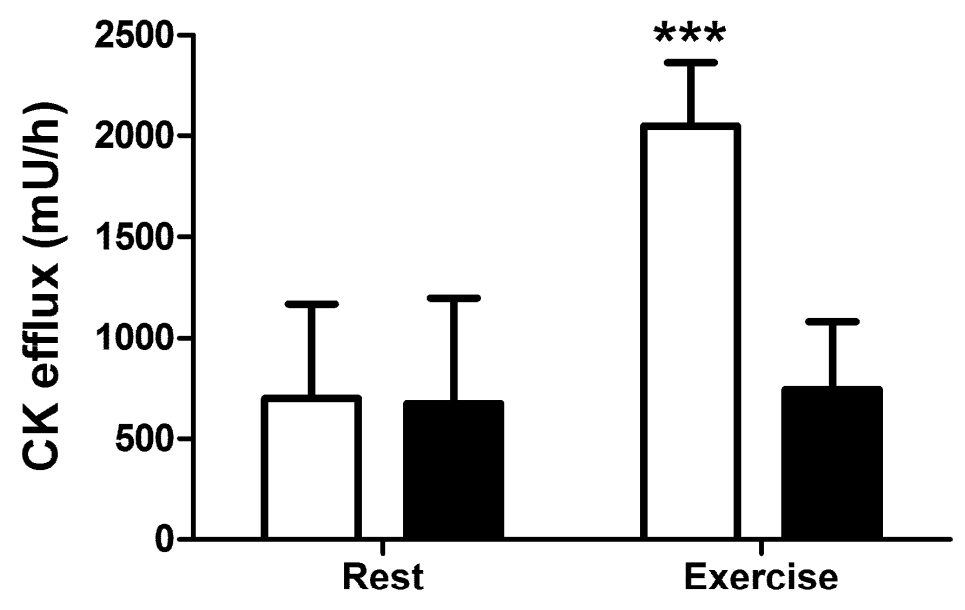

B

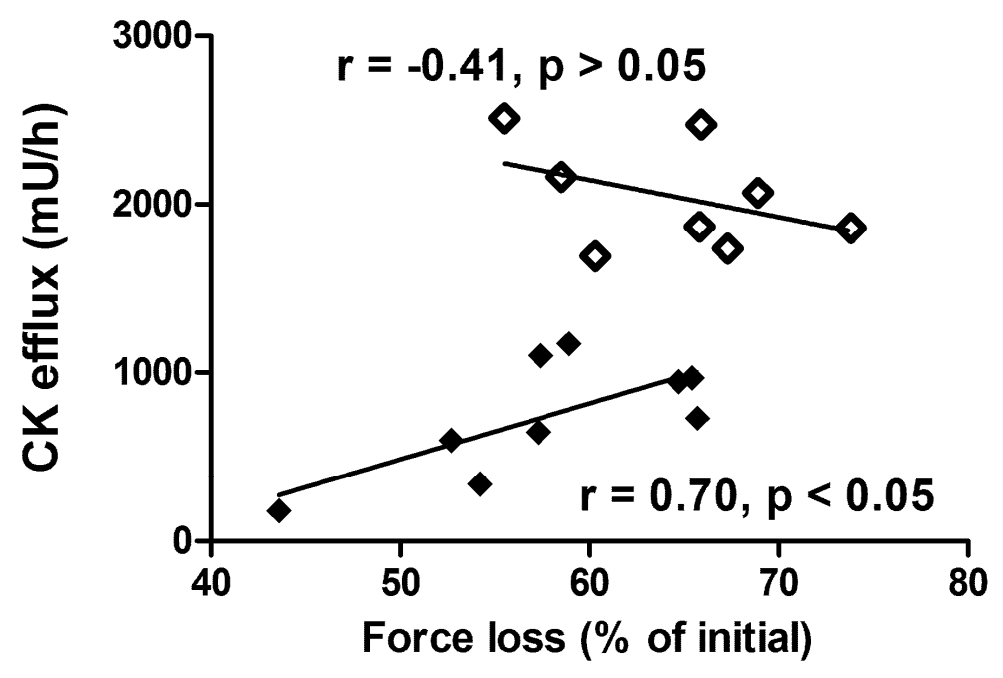

348

349 


\section{$350 \quad$ Figure captions}

351 Figure 1. Force-frequency relationship (A), twitch speed (B) and 150-Hz tetanus speed (C) in 352 control (CONT, white symbols) and regenerated (REG, black symbols) soleus muscles. * $\mathrm{P}<$ $3530.05 ; * * \mathrm{P}<0.01 ; * * * \mathrm{P}<0.001$. Values are means and S.D.

355 Figure 2. Peak isometric and eccentric force for the control (CONT, white symbols) and 356 regenerated (REG, black symbols) muscles during 100 contractions repeated every 10 s. * $\mathrm{P}<$ $357 \quad 0.05 ; * * \mathrm{P}<0.01$. Values are means and S.D.

359 Figure 3. A) Muscle CK efflux for the control (CON, white bars) and regenerated (REG, black

360 bars) muscles at rest without prior exercise and after 100 eccentric contractions; B) Scatter plot

361 of muscle CK efflux versus isometric force loss for CON muscles (white symbols) and REG

362 muscles (black symbols). Values of Pearson product-moment correlation coefficient are also

363 indicated. $* * * \mathrm{P}<0.001$. Values are means and S.D. 